\title{
Reliable Energy Sources as a Foundation for Reliable Intermittent Systems
}

\author{
Dhananjay Jagtap \\ dtjagtap@eng.ucsd.edu \\ University of California, San Diego
}

\author{
Pat Pannuto \\ ppannuto@ucsd.edu \\ University of California, San Diego
}

\begin{abstract}
This paper defines architectural and operational principles for simple and reliable energy harvesting devices that can be used in service of high-level applications. For many maintenance and monitoring tasks, we propose that it is more valuable to have reliable, trusted affirmations that nothing has changed than it is to know the exact moment that something has failed. This presents an opportunity for a new class of highly reliable, but not necessarily timely, intermittent devices. These are devices that are capable of sending messages at reasonable, fixed intervals (e.g. once an hour or once a day). They cannot activate more often, but they also promise not to activate less often. To establish this reliability, we look to opportunities for energy scavenging that are often ignored as their instantaneous power delivery capability is very limited. However, unlike higher power scavenging opportunities, many of these sources are not intermittent. Such sources may provide less than a microwatt, but their trickle of energy will be continuously, reliably available for months to decades. This enables the creation of devices that can be guaranteed to activate at predictable intervals, which allows for the construction of non-intermittent systems atop intermittent devices.
\end{abstract}

\section{CCS CONCEPTS}

- Computer systems organization $\rightarrow$ Embedded and cyberphysical systems; $\bullet$ Hardware $\rightarrow$ Batteries; $\bullet$ Human-centered computing $\rightarrow$ Ubiquitous and mobile computing.

\section{ACM Reference Format:}

Dhananjay Jagtap and Pat Pannuto. 2020. Reliable Energy Sources as a Foundation for Reliable Intermittent Systems. In The 8th International Workshop on Energy Harvesting and Energy-Neutral Sensing Systems (ENSsys '20), November 16-19, 2020, Virtual Event, Japan. ACM, New York, NY, USA, 7 pages. https://doi.org/10.1145/3417308.3430276

\section{INTRODUCTION}

With the advent of IoT, an ever-growing panoply of sensors are being conceived, constructed, and deployed to grow the reach of computational infrastructure into the physical world. Generally these devices are powered using batteries or from the mains power supply. However, we want to be able to embed intelligent sensing where there may not be mains power or where devices may not

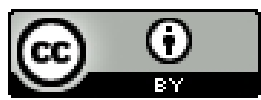

This work is licensed under a Creative Commons Attribution International 4.0 License. ENSsys '20, November 16-19, 2020, Virtual Event, Japan

(C) 2020 Copyright held by the owner/author(s).

ACM ISBN 978-1-4503-8129-1/20/11.

https://doi.org/10.1145/3417308.3430276 be accessible to change batteries [11]. This is the case when deploying sensor networks for structural or condition monitoring in industrial environments [38] or for environmental monitoring applications where there is no built infrastructure. This is part of what makes energy harvesting designs so exciting: they present the potential for set-it-and-forget it systems that can go anywhere and last forever [16].

What often makes energy harvesting designs challenging, however, is that energy sources are unreliable, unpredictable, or uncontrollable. Early designs simply ran whenever they could for as long as they could $[10,31,35]$. However, this wholly uncontrolled intermittency made it difficult for device and system designers to build applications. This has led to wide array of solutions to resolve device-level intermittency, which include hybrid and hierarchical power systems $[19,28]$, physically and logically federated energy storage banks $[1,8,15]$, and energy-aware checkpointing and energy-atomic task models $[17,26]$. While these techniques can help address and mask intermittency for applications when energy is available, to build systems of devices whose aggregate operation is reliable, we need to go back to the energy source.

Unreliably intermittent energy sources are the root cause of unpredictability for energy harvesting devices. Not all energy sources are intermittent, however. Indeed, we observe that the world is full of what we term ambient batteries. These are ambient energy sources that are able to provide similar reliability to that which is afforded to non-harvesting systems by traditional batteries. We define ambient batteries as easily harvestable sources that have deterministic and stable current and voltage characteristics over very long time periods (i.e. months to decades).

Such reliable energy would eliminate many of the historical challenges of energy harvesting systems, so why have they not been used from the beginning? It is not per lack of availability, such stable sources are shockingly prevalent (e.g. every outdoor tree, as discussed in Section 4.2.1). Notably absent from our definition of ambient batteries, however, is any minimum power delivery capability. In practice, the sources able to provide such longitudinal, reliable power may only be able to provide a microwatt or less, with only tens of millivolts of potential. It is only the recent confluence of very low power electronics and highly efficient harvesting ICs that have made such energy budgets viable for real-world systems.

At first glance, devices with an average power budget of less than one microwatt may seem very limited. As we will show in Section 4, however, when integrated over time, even a very small trickle of energy can power useful devices and applications. In particular, we focus on wide-area, longitudinal monitoring. These are challenging applications as they require large-scale deployment and must last for the lifetime of physical infrastructure. Consider the fine-grained measure of soil moisture, where topography, weather, and watering 
Table 1: Mapping Prior Applications to the General Energy Harvesting Architecture. This table looks at recent and older energy harvesting systems, and shows how the major, conceptual architecture has remained steady. One of the most interesting trends is the evolution of the energy buffer and charge controller from boutique, carefully hand-tuned circuits towards plug-'n-play, off-the-shelf components. The modularization of energy capture and management has allowed complexity to shift to the energy consumers: they have grown from transmitting fixed, 12-bit identifiers to emulating full game consoles.

\begin{tabular}{|c|c|c|c|c|}
\hline Application & Energy Source & Energy Buffer & Charge Controller & Energy Consumer \\
\hline Pushbutton (2001) [31] & Transient mechanical pulse (pushed button) & $4.4 \mu \mathrm{F}$ & MAX666 (LDO only) & Event-reporting radio \\
\hline WISP (2006) [35] & Radio waves (targeted RFID) & Capacitors (unspecified; $<1 \mathrm{mF}$ ) & Custom circuit & (Minimalist) wireless sensor node \\
\hline WATTR (2010) [6] & Variation in water pressure & $3200 \mu \mathrm{F}$ capacitor & Custom circuit & Event-reporting \\
\hline DoubleDip (2012) [28] & Temperature difference between pipe $\&$ air & ML2020 (45 mAh battery) & LTC 3109 & Sensing + event-reporting \\
\hline Monjolo $(2013)[10]$ & Magnetic field generated by wire supplying AC load & $500 \mu \mathrm{F}$ tantalum capacitor & LTC 3588 & Energy metering for AC loads \\
\hline $\begin{array}{l}\text { Battery-Free } \\
\text { Gameboy (2020) [9] }\end{array}$ & Solar energy and button presses & $3.3 \mathrm{mF}$ super capacitor & BQ25570 & Gaming console \\
\hline
\end{tabular}

influence hour-to-hour changes but erosion and climate change influence year-over-year effects. An application like this does not require tight latency; it is not important to know the exact second it started raining. But it does require reliability; it must be able to report if an area has not been watered (e.g. broken infrastructure) And it cannot require maintenance; it is costly to repeatedly send personnel to deploy and maintain sensors over large physical areas. The value from these applications is positive affirmation that nothing has changed - and nothing needs to be fixed.

We propose that the key to enabling real-world deployments for such applications is reliable intermittency. This is a primitive that addresses the intermittency of systems of intermittent devices. Prior applications-focused deployments built atop intermittent devices could not distinguish between an absence of events and broken intermittent devices [5]. To build robust, reliable applications it is equally important for devices to report when phenomena under study have not occurred as it is to report when they do.

In summary this paper makes the following contributions:

- We introduce ambient batteries, a classification for energy sources that enables reliable energy harvesting devices.

- We demonstrate how the components of energy harvesting device design have evolved to enable ambient batteries.

- We show how mitigating intermittency at the source can significantly simplify the design of energy harvesting devices.

- We introduce reliable intermittency, an abstraction that allows for reliability-oriented, wide-area systems applications.

Finally, we present a road map for new designs. We examine the edge of capability for today's technology to identify applications newly enabled by this architecture and seek to inspire new deployments of truly unattended, wide-area, ubiquitous sensing.

\section{THE DESIGN OF HARVESTING DEVICES}

In 2005, Jiang, Polastre, and Culler synthesized contemporary advancements in low-power system design and environmental energy scavenging to propose a general architecture for environmentally powered sensor networks [20]. This architecture suggested that energy harvesting devices are made of four components: an energy source, an energy buffer, a charge controller, and an energy consumer. As Table 1 shows, this architecture has withstood the test of time. To start, we look at the history and modern maturity of each of these subsystems. We suggest today it is possible to build energy harvesting devices where each subsystem is simple and reliable, which in turn allows for the creation of highly deployable devices.

We start with a short summary of the major components:

Energy Source. This provides current and potential. It can stem from a variety of physical phenomenon such as electromagnetic radiation, heat, pressure, or electrostatic charge buildup. The source refers to both the phenomenon which generates the energy and, if needed, the conversion element or transducer that converts to electrical energy that is accessible to traditional electronics frontends.

Energy Buffer. This accumulates electric charge when it is available from the source and stores it. It delivers this energy to the Energy Consumer when it is time to execute an event.

Charge Controller. This provides and maintains the desired voltage and current levels. It is responsible for energy into and out of the buffer. This is also known as Power Management Circuitry.

Energy Consumer. This is the circuit or the application that is operated from the energy provided by the source. Classical monitoring or measuring designs have three major consumers: a sensing unit, processing unit, and communication unit.

\subsection{Reconsidering Energy Sources}

Historically, energy harvesting device design has focused on everything downstream of the energy source. For device designers, there is some amount of energy available at some uncontrolled interval, which is buffered, managed, and consumed as best as possible to satisfy application goals. But why has energy harvesting design always tolerated this unreliable income, and might there be harvestable sources without such unreliability?

The popularity and categorization of today's energy sources is largely a mix of accessibility, power density, and prior successes. Sources such as solar, thermal, mechanical, and RF are readily available and apparent throughout various environments. Surveys of harvesting sources rank power density $\left(\mu \mathrm{W} / \mathrm{cm}^{3}\right)$, which directs designs towards the same, top few most capable sources [7]. These sources then develop a reputation for success, which results in better characterization of the physical phenomenon, improved conversion elements, and a body of behavioural knowledge, which in turn makes these sources easier, safer bets for future designs. 
Instead of sorting sources by power potential, we propose classifying them by the reliability of their power delivery. We suggest the following taxonomy for sources:

Impulse Sources. Impulse energy sources produce harvestable energy from an aperiodic trigger. Most commonly these are mechanical events such as pushing buttons, closing doors, human foot steps, or rubbing of two surfaces. This type of energy is available only when these event triggers occur. As a consequence, impulse sources are very poorly suited for applications which need to transmit both when an event has occurred and when it has not.

Periodic Sources. Periodic energy sources generate energy off and on for sustained windows of time. For example, outdoors, solar sources can provide up to $100 \mathrm{~mW} / \mathrm{cm}^{2}$ during peak hours, which occur for around 4-6 hours every day [13]. At night, however, they provide effectively no energy. Periodic sources are well-suited to applications that can run opportunistically, or that are able to pay the hardware complexity cost of time-shifting energy accumulation or the software complexity cost of time-shifting execution.

Constant Trickle Sources. Unlike impulse or periodic sources, constant trickle energy sources are continuously available. This means the physical phenomenon that is generating this energy is ever-present (on electronics timescales) within that environment. These are decades-long sources, such as corrosion on large, long-lived infrastructure like bridges [39]. Trickle sources may provide limited instantaneous power, but they will provide continuous power. This makes them well-suited to applications with high reliability requirements, that must be able to deliver events with certainty regardless of day-to-day environmental conditions.

In practice, constant trickle sources have been largely overlooked, likely because their power density is exceptionally low compared to impulse and periodic sources. However, the reliability and stability of trickle sources allow for the removal of income uncertainty, and the systems that manage and abstract it, from the subsequent parts of an energy harvesting designs.

\subsection{Reliability-Oriented Harvesting Design}

Reliable energy sources present the opportunity to build reliabilityoriented applications. A reliable application is one that provides continual, active affirmation about the state of the physical world.

2.2.1 Energy Sources. The key to enabling reliable devices is imposing new restrictions on the energy source. By eliminating unpredictability at the source, we can eliminate it from all downstream components. Notice that this does not presume to eliminate intermittent operation of devices, simply to make it predictable. A reliable energy harvesting device must find and utilize an ambient battery, that is, an energy source capable of providing stable, predictable, and continuous power on-demand.

2.2.2 Energy Buffer. Traditional selection criteria include total energy consumed per event, instantaneous power draw, event duration, interval between events, and total lifetime events. For simple devices, events are by definition modest in duration and draw (see Section 4.1), and the interval is permitted to be substantial. As a result, any of the common choices for the energy buffer - rechargeable batteries, supercapacitors, or tantalum capacitors - will work.
Table 2: Survey of COTS Harvester ICs. Modern harvesting chips can effectively collect energy from very limited power sources. All these chips can self-start solely from harvested energy, autonomously manage energy storage to user-configurable thresholds, and provide stable, regulated power across a range of operating voltages. There are tradeoffs between harvester choices still. Consider choosing between the LTC 3108 or ADP 5091. The ADP 5091 requires a higher cold-start voltage of $380 \mathrm{mV}$, but it offers a better efficiency of $90 \%$ at $1 \mathrm{~V}$ and $10 \mathrm{~mA}$ once running. The LTC 3108 allows for a lower cold-start voltage, but will only achieve $80 \%$ efficiency in the same steady-state conditions. For reliability-oriented design, we advocate for ample margin such that exact points on IC efficiency curves do not make-or-break successful device operation.

\begin{tabular}{c|r|r|r|r} 
Part & Cold Start & Minimum Input & Start up power & Output range \\
\hline LTC 3108 & $20 \mathrm{mV}$ & $20 \mathrm{mV}$ & $60 \mu \mathrm{W}$ & $2.35-5 \mathrm{~V}$ \\
\hline LTC 3109 & $+-1-30 \mathrm{mV}$ & $+/-30 \mathrm{mV}$ & $180 \mu \mathrm{W}$ & $2.35-5 \mathrm{~V}$ \\
\hline ADP 5091 & $380 \mathrm{mV}$ & $80 \mathrm{mV}$ & $6 \mu \mathrm{W}$ & $1.5-3.6 \mathrm{~V}$ \\
\hline S6AE101A & $2.0 \mathrm{~V}$ & $2.0-5.0 \mathrm{~V}$ & $1.2 \mu \mathrm{W}$ & $1.1-5.2 \mathrm{~V}$ \\
\hdashline BQ2550 & $600 \mathrm{mV}$ & $100 \mathrm{mV}$ & $15 \mu \mathrm{W}$ & $2-5.5 \mathrm{~V}$
\end{tabular}

The concerns that need discussion are component lifetime and sizing. Capacitors and supercapacitors support greater than a million recharge cycles from a state of deep discharge and have a very long lifetime [22]. Batteries have limited charging cycles, but can radically extend lifetime by limiting their depth of discharge [19]. As batteries generally have roughly $10 \times$ better specific energy $(\mathrm{Wh} / \mathrm{kg})$ than supercapacitors, the effective lifetime per volume becomes similar [40]. The buffer does still need to be sized large enough to satisfy the required device operation, but since this operation is expected to be nearly identical over the course of all operations, it becomes tractable to simply measure and provision [10].

2.2.3 Charge Controller. Power management requires careful manipulation of voltage and current at both the harvesting frontend and system output. This space has seen quick uptake of new circuit concepts into silicon. As seen in Table 2, today there are multiple ICs that provide rectification, boosting, power-point tracking, energy buffer management, system power management, and most recently multiple system output voltages (half of Hester's UFOP [15]). As a consequence, nearly all of the electronics complexity of harvesting is now neatly encapsulated in off-the-shelf modules. For basic energy harvesting operation, the charge controller is solved.

2.2.4 Energy Consumer. Finally, energy use must be reliable. This means devices should exhibit consistent operation (with predictable energy demand). This allows the energy buffer to be sized to allow reliable, run-to-completion semantics. It is worth highlighting that prior designs for handling on-device intermittency have shown that such simple consumption semantics do not result in optimal energy use [15, 25]. However, as Section 4.1 shows, modern low-power components leave ample headroom for simple sense-and-send operations, which allows for some inefficiency in energy use. Critically, these semantics have been demonstrated to be reliable [10] and to accelerate the design and deployment of a diverse array of devices [5]. Thus, this is a design decision motivated by our focus on wide-area, longitudinal monitoring applications that prioritizes deployability and reliability over individual device capability. 


\section{RULES OF FIVES FOR SIMPLE DEVICES}

To draw a boundary around "simple", reliable energy harvesting devices, we have developed the rules of fives. These rules are not intended as strict limits, rather they seek to provide a delineation for this class of device to enable discussion. Notice that these rules encompass the evolution of energy harvesting components, that is to say that the cost and design time argued for is only possible because of the advancements in system components outlined in the prior section. In practical application then, these rules largely guide the selection of energy sources, as that is the primary remaining source of variability for simple energy harvesting designs.

Five Places you can find it. The type of energy you intend to harvest should be widely available and readily accessible. An average person should be able to quickly think of five places where they might be able to immediately deploy a device (communication backhaul notwithstanding). The intent to exclude obscure or exotic energy sources, such as those determined by a very particular, unique geographical conditions which can be the case with geothermal energy or non-naturally occurring phenomenon like beta-voltaic radiation sources [30]. This rule also aims to add specificity. For example, "thermoelectric" sources alone is insufficient. However, "the interior versus exterior temperature gradient of any living, outdoor tree" (Section 4.2.1) meets this bar.

Five Minutes to install. The energy capture should be easy to set up. This includes the transducer or conversion element. For example, installing an earth battery is as simple as sticking two electrodes in just about any plot of land and directly attaching wires to an energy harvester. Clamping a Thermal Electric Generator to any pipe is quick and easy; scouting an area with constant airflow and clearing space for large radiator fins is not.

Five Dollar materials. The overall cost of the energy harvesting or energy conversion setup must be cheap and easy to procure. This includes the element that will either concentrate the energy or convert it. Cost determines the deployability of a system at scale.

Five Hours to design. Once a good energy source has been identified, the system should be plug-'n-play. Devices should be made of off-the-shelf components. No custom circuits or novel designs should be required to successfully collect energy. Rather, this should be something that an early-career engineer should be able to breadboard a prototype of in an afternoon. This property is core to the reliability we expect to garner from the simplicity of these designs - simple systems should use tried and tested components.

Five Events a day. The purpose of this rule is not actually to assert one specific interval. ${ }^{1}$ Rather, it aims to capture the essence of ambient monitoring applications. Namely, an energy source must provide sufficient power for a device to consistently and deterministically execute. The goal is not to instantaneously report every event; rather, the system should be the slow, steady, background heartbeat that provides validation that all is well. This design point trades latency for reliability. The principle value of these systems is checking-in over a wide area and number of instances (high deployability) that the rare event has not happened.

${ }^{1}$ Indeed, five events a week could also be appropriate. However, five per minute goes beyond ambient monitoring and five per year is too infrequent to qualify as reliable.

\section{AMBIENT BATTERIES \& APPLICATIONS}

In this section, we look to the future. We aim to quantitatively identify and characterize an array of potential ambient batteries. For each of these new potential energy sources, we also consider applications that may be well suited to their environment.

Many of these applications are motivated by reducing the maintenance burden required for long-term monitoring of wide-area environments and widely-deployed infrastructure. Historically, building such higher-level applications on top of impulse-based or certain periodic-based energy harvesting devices could not reliably distinguish whether the sensors had failed or their monitored events had simply not occurred recently. When designing applications, we want to be able to convey system information even when there is nothing "exciting" that has occurred. This means that devices must be able to reliably, periodically execute. This only possible when there is constant or periodic energy source available. To decouple application period from the period of energy sources, we focus on the class of constant trickle sources.

\subsection{Quantifying Application Energy}

Our proposed applications follow a sense-and-send archetype. This requires devices to sample the environment, perform limited computation, and communicate the result. In the spirit of simple deployments, we are excited by the potential of LP-WANs to significantly ease the communication backhaul burden. These radios tend to have higher energy requirements than more traditional low-power radios such as BLE or 802.15.4, so we consider LP-WAN transmissions a useful upper bound on communication energy needs.

In considering each of the sense, compute, and communicate steps, communication dominates the energy needs. Contemporary low power microcontrollers such as the Ambiq Micro Apollo Blue 3 require as little as $10 \mu \mathrm{A} / \mathrm{MHz}$ at $1.8 \mathrm{~V}$ [2]. In these low power modes, every 1,000 cycles of compute requires just $0.2 \mathrm{~nJ}$. Sensor energy demands are specific to application, but most of our proposed applications just require simple $\mathrm{ADC}$ reads. Using a temperature sensor as a basic example, one middle-powered option operates at $3.6 \mathrm{~V}$ and $6 \mu \mathrm{A}$ [3]. At 4 samples per second, this consumes about $22 \mu \mathrm{J}$ for one second of data. A LoRa transmission dwarfs these numbers. For an SX1272 LoRa transciever, one recent model estimated the total energy needed for one transmission is around $0.6 \mathrm{~mJ}$ (at $3.3 \mathrm{~V}$, $13 \mathrm{dBm}$ ) which would rise to around $2.6 \mathrm{~mJ}$ if maximum transmission power were used (at $3.3 \mathrm{~V}, 20 \mathrm{dBm}$ ). To compensate for the gap between models and reality, we round up and apply a conservative $10 \times$ factor and use $30 \mathrm{~mJ}$ through the rest of these applications a baseline for a single sense-and-send event. This energy estimate is further in line with preliminary testing on our own LoRa hardware.

\subsection{Some New Ambient Batteries}

We will look at several of the new possible energy harvesting sources that meet the power delivery requirements to qualify as ambient batteries. For each battery, we will consider whether it is capable of reliably powering our $30 \mathrm{~mJ}$ sense-and-send device.

4.2.1 Trees. Trees are present across urban, rural, and many unpopulated areas. The natural operation of trees provide two, independent means of establishing natural, ambient batteries. 
Tree Trunk Batteries. Remarkably, trees maintain a constant internal temperature of about $21.4^{\circ} \mathrm{C}$ regardless of the ambient temperature outside and regardless of species [14]. Trees do this by manipulating physical properties of their leaves such as changing the surface area of the leaf that exposed to the sun or allowing water to evaporate from their surface when its too hot.

Prior research has shown that the temperature gradient between tree internals and their environment can be used as an energy harvesting source $[32,36]$. This harvesting has shown consistent results in multiple locations with multiple species of trees in differnt times of year. One deployment in Paraiba, Brazil harvested from the Adenanthera pavonina (also know as the Red Lucky tree) in January 2016 [36]. Another deployment in Washington, USA harvested from a Ponderosa pine in July, 2019 [32]. Now in these two cases the climates and the species of trees both were different, however, the range of temperature variation obtained was somewhere between $4^{\circ} \mathrm{C}$ and $+5^{\circ} \mathrm{C}$ from the internal tree temperature. The average power generated was recorded to be $3.2 \mu \mathrm{W}$ [32]. Even with such a low power input, a sense and send event can be executed every 2.6 hours using our $30 \mathrm{~mJ}$ scenario.

We can optimise the design for even lower temperature changes using a LTC 3109 [4] IC. This is a dual polarity energy harvester and can work with a Ferrotec TEG [12] output of $+/-1^{\circ} \mathrm{C}$. We can harvest about $0.1 \mathrm{~mW}$ even if the temperature change is $1^{\circ} \mathrm{C}$ at $3.3 \mathrm{~V}$ [33]. Using this design we can have a sense and send event every 5 hours. Thus even with a very low temperature difference of $1^{\circ} \mathrm{C}$, there will continuously be some reliable energy available from this source. This energy harvesting could especially be useful when carrying out environmental monitoring in forests and other remote areas. It satisfies our requirements for a reliable source which is always available and ubiquitous.

Xylem Batteries. Another way of harvesting energy from trees is by using the potential difference that is generated between the Xylem of trees and the soil. This occurs mostly due to the $\mathrm{pH}$ differences between the xylem and the soil, which sets up a concentration cell of a kind and generates a potential. In this cell, the potential increases $60 \mathrm{mV}$ per $\mathrm{pH}$ unit difference and the power is available is about $0.2 \mu \mathrm{W}$ [24]. Earlier experiments demonstrated that about $0.5 \mu \mathrm{W}$ of power with a potential difference in the range of $50-230 \mathrm{mV}$ can be harvested by simply tapping an carbon, copper, or platinum electrode into the trunk of a big maple tree deep enough to be in contact with the xylem and sticking another one in the soil near the roots [18].

It is interesting to note that the 2011 study needed to design a custom energy harvester to be able to operate on such low input voltages, as well as some supporting circuitry [18]. As highlighted in Section 2.2.3, today there are many commercial energy harvester ICs that are easily available which can be directly "plugged in" to a tree and used to run low power wireless sensor nodes.

The xylem battery can power $30 \mathrm{~mJ}$ events once every 42 hours, slightly below our target. While the xylem battery does provide less power than the tree trunk battery, it also removes all the dependencies on the conversion element and the surrounding climatic variations. Even among the ambient batteries then, there is a spectrum of stability, reliability, and capability that is worth exploring.
4.2.2 Corrosion. Corrosion is process that occurs all around us. It is the degradation of refined metals into their naturally-occurring, more chemically stable oxides and sulphides. Corrosion is an electrochemical reaction that occurs due to the simultaneous reduction and oxidation of two different metals (or different surfaces of the same metal). A corrosion reaction is very similar to the reaction that occurs in conventional batteries. The value of the potential difference generated by a corrosion cell is dictated by the inherent characteristics of the metal, the relative surface areas and concentrations of the anode and cathode, the conductivity of the medium and the varying environmental conditions. These batteries provide very low instantaneous but constant power which make them an attractive option for harvesting energy. We look at two possible sources based on two different types of corrosion.

Galvanic Corrosion Battery. A galvanic series arranges metals according to the half cell potentials associated with oxidation or reduction reaction in a given environment. When two dissimilar metals are connected by an electrolyte such as water, the more electronegative metal acts as an anode and donates electrons. Sacrificial anodes, which leverage galvanic corrosion as a protection mechanism, are commonly found on bridges, buildings, hot water tanks, boats, and submerged or buried metallic structures. Unprotected metallic equipment that is in contact with water, soil, or moist air will corrode, which makes protection systems highly ubiquitous.

To understand the potential of a galvanic corrosion battery, we examine one of the most common examples: home hot water tanks (and their sacrificial anode rods). Considering the galvanic series for tap water [29], magnesium has an standard electrode potential of $-1.3 \mathrm{~V}$ while stainless steel is about $-0.05 \mathrm{~V}$, thus theoretical cell potential should be about $1.2 \mathrm{~V}$. This potential difference degrades over time and averages around $600 \mathrm{mV}$ over the entire lifetime. Magnesium has a current capacity of $1100 \mathrm{Ah} / \mathrm{kg}$, which is about $1.9 \mathrm{Ah} / \mathrm{cm}^{3}$. With tap water as the electrolyte, the current value is about $1-4 \mathrm{~mA}$. Thus, the average instantaneous power available is about $0.6 \mathrm{~mW}$. If the current draw is assumed to be $1 \mathrm{~mA}$ through its lifetime, then $1 \mathrm{~cm}^{3}$ of magnesium would take about 1,910 hours - about 80 days - to be consumed. Similarly to the xylem batteries, another nice property of galvanic corrosion cells is that the energy is already available as electrical energy and can be directly connected to an energy harvester IC. With this source we can have a sense and send event every 50 seconds.

Thermogalvanic Batteries. In the presence of different temperatures across a metal structure, very small potential differences arise. The difference in temperature causes corrosion to occur, which is referred to as either thermogalvanic corrosion or differential temperature corrosion. This phenomenon can occur across two dissimilar metals in contact or on one metallic structure with two different temperatures. This occurs most noticeably in heat exchangers or pipes carrying heated or cooled air.

Looking at heat exchangers, experiments measuring thermogalvanic corrosion observe a potential difference of around $320 \mathrm{mV}$ and a current density of $0.5 \mu \mathrm{A} / \mathrm{cm}^{2}$ across copper when both ends are maintained at $25^{\circ} \mathrm{C}$ in $\mathrm{LiBr}$ solution. This increases to about $1.5 \mu \mathrm{A} / \mathrm{cm}^{2}$ when one end is maintained at $50^{\circ} \mathrm{C}$ [37]. The instantaneous power available is between $0.48 \mu \mathrm{W} / \mathrm{cm}^{2}$ to $1.44 \mu \mathrm{W} / \mathrm{cm}^{2}$. Under the same conditions, a stainless steel Rod of AI316L grade 
will generate an instantaneous power between $0.018 \mu \mathrm{W} / \mathrm{cm}^{2}$ and $0.04 \mu \mathrm{W} / \mathrm{cm}^{2}$. Assuming a $10 \mathrm{~cm}^{2}$ surface area at room temperature, we should ideally have an instantaneous power of $4.8 \mu \mathrm{W}$ copper and $0.18 \mu \mathrm{W}$ for stainless steel. Thus, for copper we can have a sense and send event approximately every 2 hours while steel allows one event every 46 hours.

Corrosion batteries are excellent candidates for nodes that are to be placed in hard to reach places such as pipes, buildings, or in utilities-related infrastructure for structural health monitoring.

4.2.3 Earth Battery. Building an earth battery is as simple as sticking two electrodes in the ground. These electrodes can be either metals such as copper, zinc, stainless steel or non-metallic materials such as graphite. The potential difference generated between electrodes depends on a variety of phenomenon, including galvanic, microbial, and telluric effects. In practice, all of these will contribute to the overall earth battery performance.

Galvanic Effect. One study of earth batteries showed that in isolated soil, a Copper-Zinc earth battery generated about $0.9 \mathrm{~V}$ with about $0.45 \mathrm{~mA}$. That is an instantaneous power of $0.41 \mathrm{~mW}$ when separated at a distance of 250 inches. At a distance of 10 inches this current is about $0.6 \mathrm{~mA}$ which increases the instantaneous power to $0.54 \mathrm{~mW}$ [21]. Throughout the process it is noted that the potential remains constant while the current decreases. Which indicates that the earth battery potential is generated is due to the galvanic corrosion phenomenon (similar to Section 4.2.2).

Microbial Effect. The earth and the soil is home to a variety of bacteria. These bacterial colonies are responsible for breaking down complex compounds into simpler ones (decomposition). Like corrosion, this is an electrochemical process which is capable of generating an electric potential difference. Researchers have shown they can harvest about $60-100 \mu \mathrm{W}$ using soil bacteria by using Zinc and Carbon electrodes [23]. The bacterial colony grows on the carbon electrode and generates a potential difference (note: the galvanic effect will also be present in this type of cell). If instead a cell uses two carbon electrodes, which have no galvanic contribution, about $3.16 \mathrm{~mW} / \mathrm{m}^{2}$ can be harvested solely from nitrogen fixing bacteria (when the urea composition was $0.5 \mathrm{~g} / \mathrm{ml}$ of soil) [27]. This means about $3.16 \mu \mathrm{W}$ for an area of $10 \mathrm{~cm}^{2}$.

Telluric Effect. The Earth has its own magnetic field. This field is disturbed due to external interactions such as solar winds which occur regularly. This disturbance generates geo-magnetically induced currents in the Earth's crust. These low frequency currents preferentially flow from north to south, which enables well-oriented electrodes to capture energy. There is limited data on these currents, however one estimate suggests potential difference values around $0.38 \mathrm{mV} /$ feet $(1.25 \mathrm{mV} / \mathrm{m})$ [34].

Multiple phenomenons are taking place in an earth battery. As a result, this gives us an approximate range of possible instantaneous powers from $3 \mu \mathrm{W}$ to $600 \mu \mathrm{W}$. This would allow us to send a packet anywhere between once every 50 seconds to once every 3 hours. Microbial earth batteries can especially be used to our benefit in areas with a high urea content such as in farms, compost pits, or soils with large influx of industrial waste waters. These non-galvanic options are particularly of interest since the bacterial colonies are constantly regenerating, and theoretically they can last forever. The galvanic contributions are not dependent on the presence of bacteria, however, and exhibit less variation in performance from other environmental factors. In the aggregate, earth batteries satisfy all the criteria of a reliable power source.

\subsection{Ambient Batteries Are Everywhere}

We have shown an array of energy sources which cover most of the globe. These generate energy on the basis of ubiquitous phenomenon which are available continuously and will last for years if not indefinitely. We propose that it is time to start tapping the unrealized resource of reliable, ambient energy in service of reliably intermittent computing.

\section{CONCLUSIONS \& LOOKING FORWARD}

With this paper, we seek to build excitement around building "boring" sensors in the service of exciting systems. Energy harvesting and intermittent computing has historically centered on the complexities of making individual devices more capable. However, for a large number of ambient monitoring applications, on-demand, realtime, or compute-intensive sensing is simply not required. Instead, what makes these applications valuable is longevity, pervasiveness, and reliability. Maintenance can check-in every morning and know that everything on campus is okay, without needing to physically visit all of campus. City engineers can eschew the proactive costs of high-frequency human inspections, yet still be reactive to issues such as accelerated degradation of corrosion control systems, before they result in costly infrastructure failures.

We see this new direction as a triumph of the successes of intermittent computing. While there are still many interesting problems for advanced energy harvesting devices, the simple ones work pretty well today! Now that we have these robust building blocks, there is an opportunity to shift focus from the design of individual sensors to the design, operation, and capabilities of networks and systems of intermittent, energy-harvesting sensors. Let us celebrate the systems of intermittent systems engineering! It is time to see what new challenges arise with real-world deployment of systems that can be relied on by non-domain-experts to solve realworld problems, with the intent that they will run autonomously for decades. We believe that this new architecture that embraces simplicity and reliability for individual devices will be the key to unlocking rich, reliable, and pervasive systems.

\section{REFERENCES}

[1] J. Adkins, B. Ghena, N. Jackson, P. Pannuto, S. Rohrer, B. Campbell, and P. Dutta. The signpost platform for city-scale sensing. In Proceedings of the 17th ACM/IEEE International Conference on Information Processing in Sensor Networks, IPSN'18, New York, NY, USA, April 2018. ACM.

[2] Ambiq Micro. Apollo 3 Blue Plus MCU datasheet. https://ambiq.com/apollo3blue-plus/\#documents, 2020.

[3] ams AG. AS621X Digital temperature sensor. ams.com/documents/20143/36005/ AS621x_DS000677_2-00.pdf/a90b32a5-d1f8-e6df-5327-bec856e093dd, 2020.

[4] Analog Devices. LTC 3109. https://www.analog.com/media/en/technicaldocumentation/data-sheets/3109fb.pdf.

[5] B. Campbell and P. Dutta. An energy-harvesting sensor architecture and toolkit for building monitoring and event detection. In Proceedings of the 1st ACM Conference on Embedded Systems for Energy-Efficient Buildings, BuildSys'14, pages 100-109, New York, NY, USA, nov 2014. ACM.

[6] T. Campbell, E. Larson, G. Cohn, J. Froehlich, R. Alcaide, and S. N. Patel. WATTR: A method for self-powered wireless sensing of water activity in the home. In 
Proceedings of the 12th ACM International Conference on Ubiquitous Computing, UbiComp '10, page 169-172. Association for Computing Machinery, 2010.

[7] S. Chalasani and J. M. Conrad. A survey of energy harvesting sources for embedded systems. In IEEE SoutheastCon 2008, pages 442-447, 2008.

[8] A. Colin, E. Ruppel, and B. Lucia. A reconfigurable energy storage architecture for energy-harvesting devices. SIGPLAN Not., 53(2):767-781, Mar. 2018.

[9] J. de Winkel, V. Kortbeek, J. Hester, and P. Pawełczak. Battery-free game boy. In Proceedings of the ACM on Interactive, Mobile, Wearable and Ubiquitous Technologies, Sepetember 2020

[10] S. DeBruin, B. Campbell, and P. Dutta. Monjolo: An energy-harvesting energy meter architecture. In 11th ACM Conference on Embedded Networked Sensor Systems (SenSys '13), 2013.

[11] B. Denby and B. Lucia. Orbital edge computing: Nanosatellite constellations as a new class of computer system. In Proceedings of the Twenty-Fifth International Conference on Architectural Support for Programming Languages and Operating Systems, ASPLOS '20, page 939-954, New York, NY, USA, 2020. Association for Computing Machinery.

[12] FerroTec. Peltier cooler model 9500/127/100 B. https://thermal.ferrotec.com/ products/peltier-thermoelectric-cooler-modules/9500 127 100-b/.

[13] M. Habibzadeh, M. Hassanalieragh, A. Ishikawa, T. Soyata, and G. Sharma. Hybrid solar-wind energy harvesting for embedded applications: Supercapacitor-based system architectures and design tradeoffs. IEEE Circuits and Systems Magazine, 17(4):29-63, 2017

[14] B. R. Helliker and S. L. Richter. Subtropical to boreal convergence of tree-leaf temperatures. Nature, 454, July 2008.

[15] J. Hester, L. Sitanayah, and J. Sorber. Tragedy of the Coulombs: Federating energy storage for tiny, intermittently-powered sensors. SenSys '15, 2015.

[16] J. Hester and J. Sorber. The future of sensing is batteryless, intermittent, and awesome. SenSys '17, 2017.

[17] J. Hester, K. Storer, and J. Sorber. Timely execution on intermittently powered batteryless sensors. In Proceedings of the 15th ACM Conference on Embedded Network Sensor Systems, SenSys '17, New York, NY, USA, 2017. Association for Computing Machinery.

[18] C. Himes, E. Carlson, R. J. Ricchiuti, B. P. Otis, and B. A. Parviz. Ultralow voltage nanoelectronics powered directly, and solely, from a tree. VOL. 9, NO. 1, January 2010.

[19] N. Jackson, J. Adkins, and P. Dutta. Capacity over capacitance for reliable energy harvesting sensors. In The 18th International Conference on Information Processing in Sensor Networks, IPSN'19. ACM, April 2019.

[20] X. Jiang, J. Polastre, and D. Culler. Perpetual environmentally powered senso network. In IPSN 2005. Fourth International Symposium on Information Processing in Sensor Networks, 2005, 2005

[21] N. Khan, Z. Saleem, and N. Abas. Experimental study of earth batteries. In Second International Conference on Electrical Engineering, 2008.

[22] J. Libich, J. Máca, J. Vondrák, O. Čech, and M. Sedlaříková. Supercapacitors Properties and applications. Journal of Energy Storage, March 2018.

[23] F. Lin, Y. Kuo, J. Hsieh, H. Tsai, Y. Liao, and H. Lee. A self-powering wireless environment monitoring system using soil energy. IEEE Sensors fournal, 15(7):3751-3758, 2015

[24] C. J. Love, S. Zhang, and A. Mershin. Source of sustained voltage difference between the xylem of a potted ficus benjamina tree and its soil. PLos One, 3 , August 2008.

[25] B. Lucia, V. Balaji, A. Colin, K. Maeng, and E. Ruppel. Intermittent Computing Challenges and Opportunities. In B. S. Lerner, R. Bodík, and S. Krishnamurthi, editors, 2nd Summit on Advances in Programming Languages (SNAPL 2017), volume 71 of Leibniz International Proceedings in Informatics (LIPIcs), pages 8:1-8:14, Dagstuhl, Germany, 2017. Schloss Dagstuhl-Leibniz-Zentrum fuer Informatik.

[26] K. Maeng and B. Lucia. Adaptive dynamic checkpointing for safe efficient intermittent computing. In Proceedings of the 13th USENIX Conference on Operating Systems Design and Implementation, OSDI'18, page 129-144, USA, 2018. USENIX Association.

[27] V. Magotra and S. K. T. e. a. Kumar. Compost soil microbial fuel cell to generate power using urea as fuel. Nature, 4154, March 2020.

[28] P. Martin, Z. Charbiwala, and M. Srivastava. DoubleDip: Leveraging thermoelectric harvesting for low power monitoring of sporadic water use. In Proceedings of the 10th ACM Conference on Embedded Network Sensor Systems, SenSys '12, page 225-238, New York, NY, USA, 2012. Association for Computing Machinery.

[29] Y. Matsukawa, H. Chuta, M. Miyashita, M. Yoshikawa, Y. Miyata, and S. Asakura. Galvanic series of metals conventionally used in tap water with and without flow and its comparison to that in seawater. CORROSION, 67(12), 2011.

[30] L. Olsen, P. Cabauy, and B. Elkind. Betavoltaic power sources. Physics Today V65, December 2012.

[31] J. A. Paradiso and M. Feldmeier. A compact, wireless, self-powered pushbutton controller. In International Conference on Ubiquitous Computing, pages 299-304. Springer, 2001.

[32] N. Purcell, J. Stevens, Carlson.E, G. Boyer, and O. Baiocchi. Harvesting energy from tree trunks. June 2019
[33] D. Salerno. Ultra-low voltage energy harvester uses thermoelectric generator for battery-free wireless sensors. 2011

[34] A. L. Smart. The Trans Alaska Pipeline - Potential measurements and telluric current. IEEE Transactions on Industry Applications, IA-18(5):557-567, 1982.

[35] J. R. Smith, A. P. Sample, P. S. Powledge, S. Roy, and A. Mamishev. A wirelesslypowered platform for sensing and computation. In P. Dourish and A. Friday, editors, UbiComp 2006: Ubiquitous Computing, pages 495-506, Berlin, Heidelberg, 2006. Springer Berlin Heidelberg.

[36] C. Souza, F. Carvalho, F. Silva, H. Andrade, N. Silva, O. Baiocchi, and I. Müller. On harvesting energy from tree trunks for environmental monitoring. International Journal of Distributed Sensor Networks, 12, June 2016.

[37] R. Sánchez-Tovar, M. Montañés, and J. García-Antón. Thermogalvanic corrosion and galvanic effects of copper and AISI 316L stainless steel pairs in heavy LiBr brines under hydrodynamic conditions. Corrosion Science, 60:118-128, 2012.

[38] X. Tang, X. Wang, R. Cattley, F. Gu, and A. D. Ball. Energy harvesting technologies for achieving self-powered wireless sensor networks in machine condition monitoring: A review. Sensors(Basel), 18(12), December 2018.

[39] Transportation Research Board and National Academies of Sciences, Engineering, and Medicine. Cathodic Protection for Life Extension of Existing Reinforced Concrete Bridge Elements. The National Academies Press, Washington, DC, 2009.

[40] J. Xie, P. Yang, Y. Wang, T. Qi, Y. Lei, and C. M. Li. Puzzles and confusions in supercapacitor and battery: Theory and solutions. Fournal of Power Sources, 401:213-223, 2018 